\title{
A Composition of RDFL and XML-DOM for Improving the Efficiency of Semantic Search
}

\author{
K. Sangeetha \\ Assistant Professor, CSE, M.Kumarsamy College of Engineering (Autonomous), \\ Karur, Tamilnadu \\ S. Saravanan \\ Assistant Professor ,Department of Computer Science and Engineering, \\ M. Kumarasamy College of Engineering, Karur, Tamil Nadu, India
}

\begin{abstract}
In the Present World, the practice on Internet is augmented in an extremely speedy way. The Person who is using who is using the Internet all the time needs to dig out the consequential knowledge from the possessions. When the count of person who using is increased, the Internet is dispersed gradually. In order to avoid this, Semantic Technology like RDL beside by XML DOM were there to fetch obvious responses by using queries by intriguing assistance of the definite semantic knowledge perspective of the search engines.
\end{abstract}

Keywords:, Resource Description Language, Semantic Technology XML DOM, Segment Fusing, Precedence Algorithm.

\section{INTRODUCTION}

The implausible development of Web presents an effortless mode for accessing the knowledge. Internet is one of the partially-structured documents where it is there to provide an enormous number of knowledge. Up-to now, the majority of the conventional search engine gets the knowledge syntactically accurate but it is large in amount. Not barely used for establishing the applicable knowledge precisely but as well used to way in the variety of knowledge from plentiful possessions. Extraordinary escalation in the data facts and web facts within the conventional search engines are not appropriate to any further extent.

The Semantic Net is not anything except the conservatory of the up to date Internet in which it offers the knowledge extra persuasive. Nowadays, the semantic engine is utilized to erect the inside of Internet comprehensible just to peoples and not meant for the technology.

By then, the Semantic Internet technology occupies an important role in enlarging the inside of Internet to be explicable by the machines. A realistic assessment on Semantic Internet function recognized the 4 main confronts for implementing the majority of common functionality related to Semantic Internet technologies from a software engineering point of view:

(1) The concern involved in incorporating dusty and different types of data.

(2) The difference of facts and APIs involving works.

(3) Undeveloped tardy best practices and standards.

(4) Allocation of application logic across components.

Categorizing these disputes permits the recovered assessment of the expenses associated with assuming Semantic Internet technologies inside activity, and outlines the base for deceitful enhanced architecture for utilizing the Internet of Data. 


\section{ASSOCIATED DATA ON SEMANTIC SEARCH}

The Succession of Semantic Internet technologies are classified by the determined starter of novel thoughts, principles, and knowledge's. Through assessing the WWW with Semantic Internet, it is extremely apparent to facilitate the Semantic Internet is not a replacement of World Wide Internet, but it's an enhancement of the current Internet.

During current existence, major Internet data sources such as Msn, Google, Yahoo, eBay and Amazon have started their progress in providing admittance to their records through Internet APIs. But the difficulty in the standard Internet APIs is that, it does not allocate worldwide sole identifiers to data items, where it is not likely to set hyperlinks between data items provided by different APIs.

In order to overcome that and to offer a single Internet of Data, suppliers want to go after the shared policy to distribute their data and connect it to other data sources.

\section{RESOURCE DESCRIPTION LANGUAGE}

The Resource Description Language (RDFL) is one of the entity of World Wide Internet Consortium (W3C) where it is mainly used to encode the meaning of the data. Also, it is in the form of metadata. This Resource Description Language plays an important role in the place of the knowledge about the internet resources.

Basic form is,

\section{Sub Pro Obj .}

Multiple Properties and Objects are represented as,

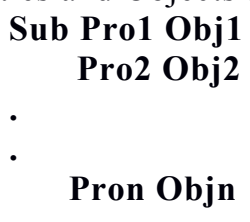

Pron Objn

The building block in RDFL is an Obj-Att-Val triple, can write as Att (Obj, Val)

[Obj]-Att[Val]

\section{XML DOCUMENT OBJECT MODEL}

The XMLDOM be the establishment of XML. XML papers include a ladder of knowledgeable item known as nodes; XMLDOM be a method of recitationing nodes along with the associations among them.

\section{CONVENTIONAL INTERNETS}

The conventional Internet goes behind the worldwide record that has not in the existence of semantic structure. Some of the limitations are,

- Internet substance requires an appropriate formation concerning the depiction of knowledge.

- Uncertainty of knowledge leads to meager incorporation of knowledge.

- Incapable to ensuring trust at all levels.

- Inability by the lack of a universal format.

- Habitual knowledge relocate is deficient. 


\section{PROJECTED IDEA}

Here in this suggested idea, the RDFL and XMLDOM engage in recreation wherever the contraption can appreciate the connotation with the known statement throughout its triple pattern. It comprises two kind of procedure to be passed out to bring the relevancy data.

\section{RDFL and XMLDOM}

In RDFL, two ways is used to search. They are, by developing the tools and applications which routinely embed RDFL Meta data in documents and other is generated by record and presented on dynamic Internet page's which can be easily supplemented with metadata and RDFL statements, expressing the exact meaning of the data originally coming from the fields of the database table.

It comprises of,

o Person who is using Query,

o RDFL translation,

o Other Search engines,

o User Query Results,

o RDFL Process,

o XMLDOM Process,

o Normalization

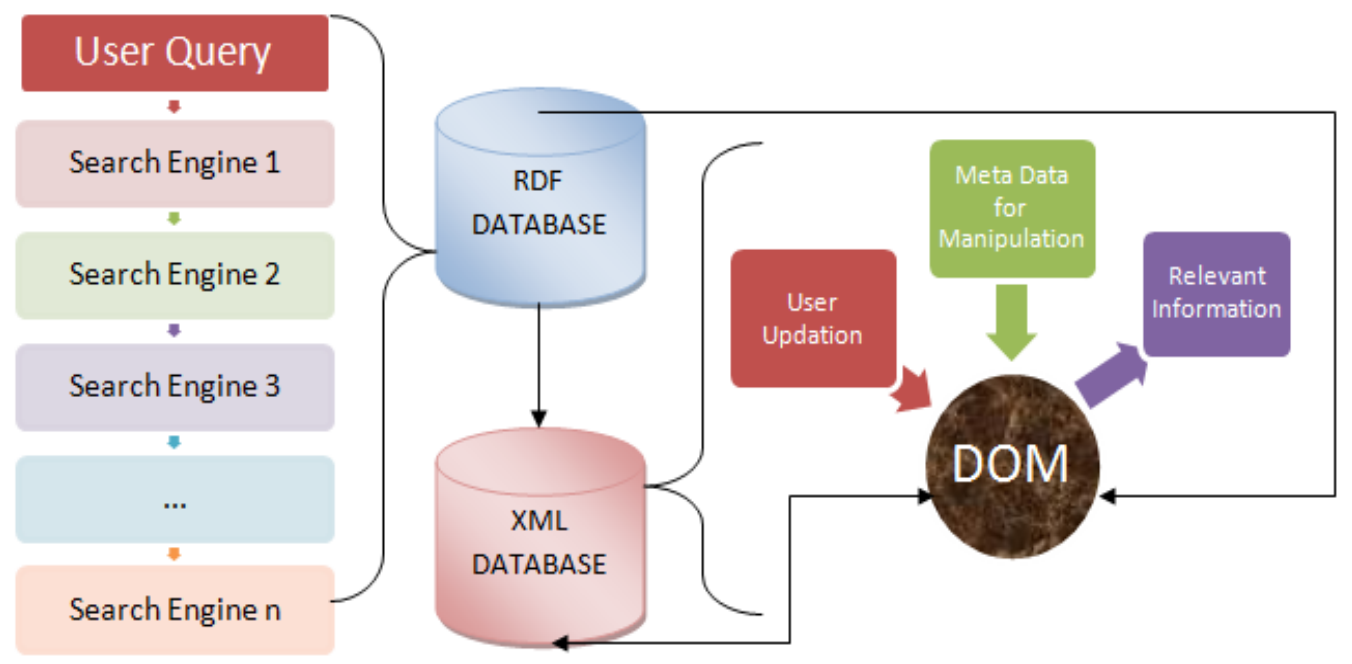

Figure 1: System Design.

\subsection{RDFL translation}

The RDFL translation is employed with RDFL where it contains the Sub, Obj and Pre.

A structured of RDFL in Translation is an Obj-Att-Val triple, were written as Att (Ob, Val) 


\subsection{Other Search engine}

In this, the query which was given by person who is using is looked for the knowledge in two ways. First way is to search the other Internet sites by swarming pertinent to inquiry sub uncertainty and dig out the applicable data's from the other Internet sites. Second way, is to refer the inner database. These both the things and works were done by the Parsing algorithm where it parses the explored substance of those search engine.

\subsection{Person who is using Query Results}

It is used to identify the knowledge that is related to the person who is using query. It creep the real content of the person who is using query that is placed in various Internet sites. By swarming those related knowledge it supplies the Internet site, it can be done by the algorithms.

\subsection{RDFL Process}

It is used as a snatch where each snippet includes a casement of selected text from a manuscript that embraces the wordings of the queries.

\section{A1: RDFL Algorithm}

Let us consider a inquiry comprising of $r$ keywords ka1... kan. Let $M=\{1 \ldots n\}$ and $S 0=\{e \mid e \in E q M$ contains(e, kai) \}. Two Sets related to this A2 algorithm are,

a) $\{\mathrm{e} \mid \mathrm{e} \in \mathrm{S} 0 \wedge \mathrm{p}(\mathrm{e}, \mathrm{p}) \in \mathrm{CsE}=>\mathrm{p} \in \mathrm{S} 0\}$

b) $\left\{\mathrm{e} \mid \mathrm{p}\left((\mathrm{e}, \mathrm{p}) \in \mathrm{CsE}^{\wedge} \mathrm{p} \in \mathrm{S} 0\right\}\right.$

\subsection{XMLDOM Process}

\section{A 2: XMLDOM Fragment Fusing Algorithm}

Comparison of Words:

$$
\begin{gathered}
\mathrm{Pa} 1[\mathrm{Ca}-2]=\mathrm{CaSa} / \mathrm{Sa}[\mathrm{Ja}-1] \\
\mathrm{Pa}=\mathrm{Pa}+\mathrm{Pa} 1[\mathrm{Ca}-2]
\end{gathered}
$$

Probability:

$$
15.0 * \mathrm{MPa}(3.0,(\mathrm{U}+2)-2.0)-6
$$

\subsection{Normalization}

The result of enchanting away the Maximized value by the Minimized value.

where

$$
\mathrm{Pa} 1[\mathrm{Ga}]=(\mathrm{Pa}[\mathrm{Ga}]-\mathrm{MINa}) /(\mathrm{MAXa}-\mathrm{MINa})
$$

$\mathrm{Pa}[\mathrm{Ga}], \mathrm{Pa} 1[\mathrm{Ga}]=$ Probability Numbers,

As a result, the ultimate seek knowledge can be recognized by means of these algorithms and priority.

\section{CONCLUSION AND FUTURE ENHANCEMENT}

This proposed system concludes by the importance and consequences of RDFL Search and XMLDOM Search that had the propensity on augment with Conventional Internet with tenacity towards the confines are exposed. It also formulates the system to be aware of knowledge's, where it fetches the applicable knowledge's all way through the 
Uniform Resource Identifiers. Consequently user who is performing their search is clever toward identifying their most pertinent knowledge's by commencing these explorations. In the future enhancement, there will be an improvement of this planned proposed system together with modern archetype along with industrialized systems.

\section{REFERENCES}

[1] Apparao.V, et al., Editors. World Wide Internet Consortium, http: //www. w3. org /TR/1998/REC-XMLDOM-Level-1-19981001.

[2] Benjamin Heitmann, Richard Cyganiak, Conor Hayes, Stefen Decker, "An Empirical Grounded Conceptual Architecture for Applications on the Internet of Data", IEEE TRANSACTION ON SYSTEMS, MAN, AND CYPERNETICS, Vol. 42, No.1, 2012, ISSN: $1094-6977$.

[3] Benjamin Heitmann, Sheila Kinsella, Conor Hayes, Stefen Decker, "Implementing Semantic Internet applications: reference architecture and challenges".

[4] Frank Van Harmellen, Dieter Fensel, Michel Klein, Jeen Brokstra, “ A Internet-Search Engine based approach to measure semantic similarity between words“,IEEE TRANSACTION ON KNOWLEDGE AND ENGINEERING,ISSN: 1041-4347/11.

[5] Maire Casey, Claus Pahl, "Internet Components and the Semantic Internet".

[6] T. Neumann and G. Moerkotte. Characteristic sets: Accurate cardinality estimation for RDFL queries with multiple joins. In ICDE, pages 984 \{994. IEEE Computer Society, 2011.

[7] T. Neumann and G. Weikum. The RDFL-3X engine for scalable management of RDFL data. VLDB J.,19(1):91 \{113, 2010.

[8] B. Quilitz and U. Leser. Querying distributed RDFL data sources with SPARQL. In ESWC, pages 524\{538. Springer, 2008.

[9] RDFL primer. http://www.w3.org/TR/RDFL-primer/.

[10] RDFL vocabulary description language 1.0

[11] Raul Garcia-Castro, Asuncion Gomez- Perez, Oscar Munzon-Garcia, "The Semantic Internet Framework:a component-based framework for the development of Semantic Internet applications".

[12] A. Schmidt, et al., "The XML Benchmark Project", Tech.Report INS-R0103, CWI, The Netherlands, 2001.

[13] L. Sidirourgos, R. Goncalves, M. L. Kersten, N. Nes, and S. Manegold. Column-store support for RDFL data management: not all swans are white.

[14] Thilagamani, S. and N. Shanthi, 2010. Literature survey on enhancing cluster quality. Int. J. Comput. Sci. Eng.., 2: $1999-2002$. http://www.enggjournals.com/ijcse/doc/IJCSE10- 02-06-26.pdf

[15] S. Chitra, B. Madhusudhanan, G. Sakthidharan, P. Saravanan, Local Minima Jump PSO for Workflow Scheduling in Cloud Computing Environments, Springer, ISBN 364241673X, 1225-1234, 2014.

[16] E.T. Venkatesh, P. Thangaraj, and S. Chitra, " An Improved Neural Approach for Malignant and Normal Colon Tissue Classification from Oligonucleotide Arrays ,” European J. Scientific Research , vol. 54, $\quad$ pp. 159-164, 2011 